\title{
ChemComm
}

Check for updates

Cite this: Chem. Commun., 2019,

55, 3239

Received 30th October 2018

Accepted 31st January 2019

DOI: $10.1039 / \mathrm{c} 8 \mathrm{cc} 08670 \mathrm{~b}$

rsc.li/chemcomm

\section{Directed evolution of the optoelectronic properties of synthetic nanomaterials $\dagger$}

\author{
Benjamin Lambert, (D) Alice J. Gillen, (D) Nils Schuergers, (D) Shang-Jung Wu (D) and \\ Ardemis A. Boghossian (D) *
}

\begin{abstract}
Directed evolution is a powerful approach to tailor protein properties toward new or enhanced functions. Herein, we use directed evolution to engineer the optoelectronic properties of DNA-wrapped singlewalled carbon nanotube sensors through DNA mutation. This approach leads to an improvement in the fluorescence intensity of $56 \%$ following two evolution cycles.
\end{abstract}

Synthetic nanomaterials are typically known to rely on a rational approach for tuning their physical and chemical properties. This approach involves modifying the morphology, composition, shape, size, or surface chemistry of the material in a predictive manner to achieve a desired outcome. ${ }^{1-3}$ For instance, the optoelectronic properties of quantum dots can be readily tuned by changing particle size. ${ }^{4}$ Nevertheless, this rational approach is limited to materials for which the relationship between material's structure and function is known.

Materials lacking a defined structure-function relationship are commonly encountered in the field of protein engineering. To engineer these biomaterials, directed evolution, which relies on the iterative screening and selection of mutants from a collection of mutated proteins, is used to enhance or change a desired protein function. ${ }^{5,6}$ Herein, we apply a directed evolution approach to engineer the optoelectronic properties of nanomaterials, in particular single-stranded DNA-wrapped single-walled carbon nanotubes (ssDNA-SWCNTs) complexes, which currently lack a defined structure-function relationship.

SWCNTs are cylindrical one-dimensional nanostructures that can be conceptualised as rolled-up sheets of graphene. ${ }^{7}$ The rolling angle of the graphene sheet determines the $(n, m)$ chirality of the SWCNT, resulting in either metallic or semiconducting properties. ${ }^{7}$ Semiconducting SWCNTs have attracted particular interest in the field of optical biosensing owing to

Institute of Chemical Sciences and Engineering (ISIC), Ecole Polytechnique Fédérale de Lausanne (EPFL), 1015-Lausanne, Switzerland.

E-mail: ardemis.boghossian@epfl.ch

$\dagger$ Electronic supplementary information (ESI) available. See DOI: 10.1039/ c8cc08670b their near-infrared emission that presents many advantages over the emissions of conventional fluorophores. These advantages include fluorescence tunability and long-term photostability, as well as the ability to image in living tissue and blood, which absorb minimally in the near-infrared range. ${ }^{8-10}$ Furthermore, the surface of SWCNTs can be functionalised with biomolecules such as ssDNA. This functionalisation imparts both colloidal stability in aqueous solutions and analyte specificity for optical sensing applications. ${ }^{11-13}$ Previous studies have reported that the sequence of the DNA used to suspend the nanotube contributes to variations with respect to SWCNT chirality affinity, analyte selectivity, and the fluorescence intensity of the ssDNASWCNT complex. ${ }^{14-18}$ However, a general understanding of how the DNA sequence affects these properties is lacking.

An empirical approach has thus far been used to identify ssDNA sequences that yield complexes with a preferred fluorescence responses to specific analytes. ${ }^{13,16}$ In this approach, SWCNTs wrapped with different oligomers are incubated in the presence of various analytes and specificity is determined according to the observed fluorescence response. Sensors developed using this approach are currently used for in vitro and in vivo measurements. ${ }^{19-21}$ However, these sensors suffer from limited quantum yields (QYs), typically lower than surfactantsuspended SWCNTs which report maximum QYs of $\sim 1 \%$. $^{22-25}$ In addition, this current approach has been used to monitor the response of only 13 distinct DNA oligomers at a time. Given that a typical DNA sequence used in these studies is approximately 30 nucleotides long, these oligomers have a total of $4^{30}$ different possible sequence combinations. As a result, the performance of the majority of these distinct ssDNA-SWCNT sensors is overlooked when using the current approach.

Directed evolution offers a guided search through this vast DNA sequence space. In this article, we use directed evolution to increase the fluorescence efficiency of a sSDNA-SWCNT sensor. The evolution of the ssDNA-SWCNT sensor was done through the cyclic procedure illustrated in Fig. 1a: (1) random mutation of a selected sensing oligomer; (2) wrapping of the mutated oligomers on SWCNTs; (3) screening of the SSDNA-SWCNT 
a)

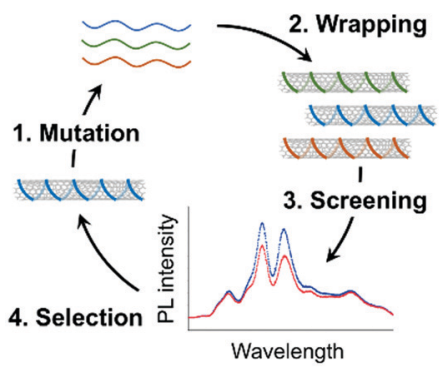

b)

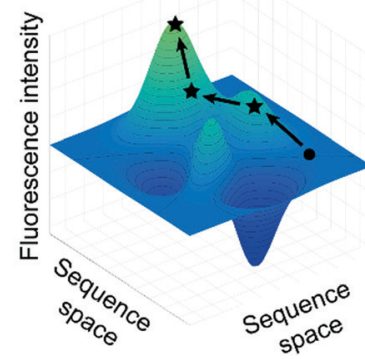

Fig. 1 Application of directed evolution to ssDNA-SWCNTs. (a) Scheme of an evolution cycle for ssDNA-SWCNTs. Various ssDNA sequences are wrapped on SWCNTs, and the library of sSDNA-SWCNTs is screened for a desired property such as increased fluorescence intensity. (b) Graphical representation of a hypothetical fitness landscape for sSDNA-SWCNTs. This plot illustrates how a particular property of the complexes may be evolved through ssDNA mutation. The fitness, in this case fluorescence intensity, of the sensors is represented as a function of the different DNA sequences. A representative evolution pathway is shown in black, where the circle represents the starting complex, and the stars represent the successive, improved mutants obtained after each round of screening.

complexes for enhanced fluorescence; and (4) selection of the mutants showing the highest fluorescence intensity for the next round of mutation. This approach assumes a smooth fitness landscape (Fig. 1b) whereby the evolution of a particular ssDNA-SWCNT property, in this case the fluorescence intensity, is envisioned as a continuous path among peaks and valleys which represent the respective enhancement or attenuation of this property.

In this work, we applied a directed evolution approach to enhance the fluorescence intensity of ssDNA-SWCNT variants based on the $(\mathrm{GT})_{15}$ sequence. The (GT) ${ }_{15}$ sequence was chosen because of its application in neurotransmitter sensing. ${ }^{16}$ Three random nucleotide substitutions were introduced computationally at randomized locations within the sequence (ESI $\dagger$ ). These randomly generated $(\mathrm{GT})_{15}$ derivative sequences were chemically synthesized (Microsynth AG, Switzerland) with a $>99.25 \%$ coupling efficiency. For the first evolution cycle, 99 mutants of the $(\mathrm{GT})_{15}$ sequence were screened for increased integrated fluorescence intensity and for increased intensity of individual SWCNT chiralities. A second round of mutagenesis was performed by introducing three random nucleotide substitutions to the mutants exhibiting increased integrated fluorescence intensity. For this round, a library size of only 10 additional sequences was needed to identify additional mutants that exhibited greater fluorescence enhancement. The absorbance and fluorescence of the SWCNT complexes were compared before and after wrapping to verify that the nanotubes were well suspended by the ssDNA (Fig. S1, S2 and S6, ESI†).

We identified several mutants in each cycle that exhibited higher fluorescence intensities compared to the starting (GT) ${ }_{15}$-SWCNT complex (Fig. 2a and Table 1). The sequences of the DNA mutants are provided in Table S1 (ESI $\dagger$ ). In the first mutagenesis cycle, we identified two mutant complexes that demonstrated statistically increased integrated fluorescence

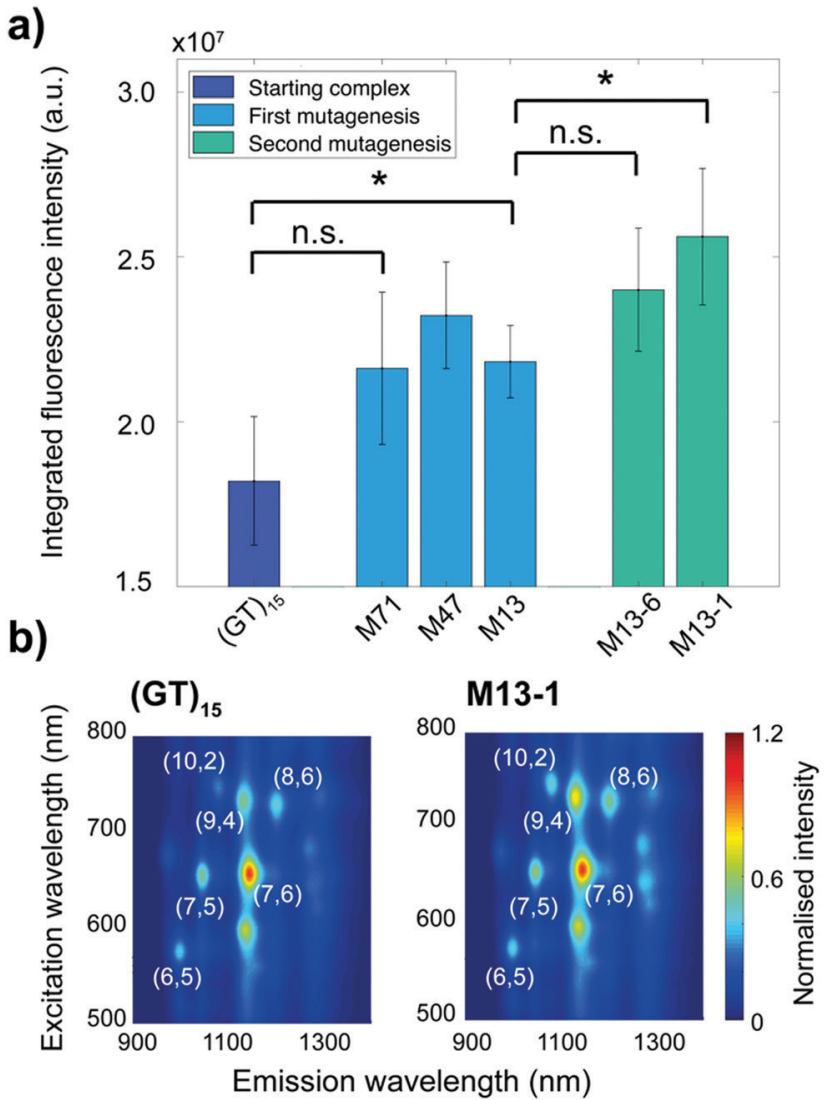

Fig. 2 Increase of fluorescence intensity after two evolution cycles. (a) Integrated fluorescence intensity under $745 \mathrm{~nm}$ excitation of the ssDNA-SWCNT mutants normalised by the (GT) ${ }_{15}$-SWCNT intensity. The integrated intensity is calculated from the additive emissions for wavelengths between 900 and $1400 \mathrm{~nm}$. The error bars represent 1 standard deviation ( $n=3$ replicates). The * symbol indicates $p<0.05$ and "n.s." indicates $p>0.05$ according to two-sample student $t$-tests. (b) Photoluminescence excitation (PLE) maps of the (GT) ${ }_{15}$-SWCNT and M13-1-SWCNT complexes. The SWCNT chiral indices $(n, m)$ are indicated in white. The fluorescence intensities are normalised to the maximum intensity in each plot.

intensity compared to the (GT) ${ }_{15}$-SWCNT complex: the M13and M47-SWCNT mutants (Fig. 2a). The second mutagenesis cycle, that was performed on the M13 sequence, yielded two additional mutants, M13-1- and M13-6-SWCNT, that showed increased integrated fluorescence intensity compared to $(\mathrm{GT})_{15^{-}}$ SWCNT complex. Furthermore, the M13-1-SWCNT mutant showed enhanced fluorescence compared to the M13-SWCNT mutant.

In addition to increases in integrated fluorescence emissions, we noted a strong chirality dependence on fluorescence enhancement. As shown in the photoluminescence excitation maps (Fig. 2b), the increase in fluorescence intensity was larger for the $(9,4),(10,2)$, and $(8,6)$ chiralities. A quantitative comparison of the fluorescence changes for the different chiralities and the integrated intensity relative to the starting $(\mathrm{GT})_{15}$-SWCNT complex is shown in Table 1. Overall, the M13-1-SWCNT showed the greatest fluorescence enhancement with an improvement in the integrated intensity of $41 \%$ compared to $(\mathrm{GT})_{15}$-SWCNT. Moreover, the $(9,4),(10,2)$, and $(8,6)$ chiralities showed fluorescence intensity increases of up to $43 \%, 54 \%$, and $56 \%$, respectively. 
Table 1 Fluorescence intensity change (in \%) for the ssDNA-SWCNT mutants compared to the $(G T)_{15}$-SWCNT complex for the $(6,5),(7,5),(7,6),(9,4)$, $(10,2)$ and $(8,6)$ chiralities. The integrated fluorescence intensity under $745 \mathrm{~nm}$ excitation is labelled as "ALL"

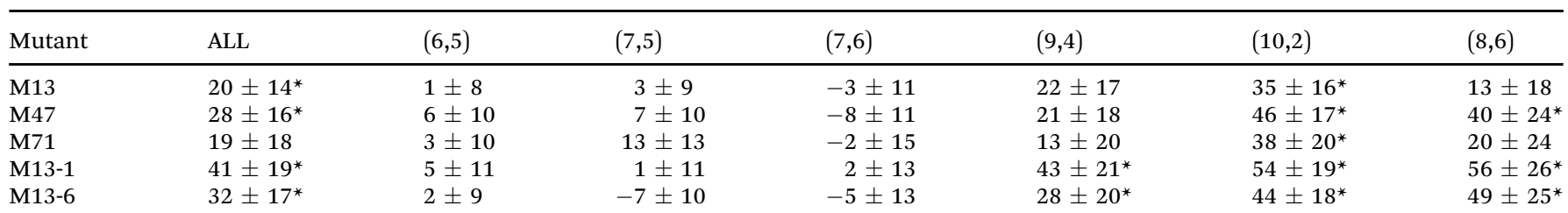

The error represents 1 standard deviation ( $n=3$ replicates). The * symbol indicates $p<0.05$ for two-sample student $t$-tests between the complex and $(\mathrm{GT})_{15}$-SWCNT.

Although the increase in the integrated intensity of the M71-SWCNT was not statistically significant compared to $(\mathrm{GT})_{15}$-SWCNT (Fig. 2a), significant increases (+38\%) were observed for the $(10,2)$ chirality (Table 1). Additional measurements involving surfactant replacement of the SSDNA $^{18}$ verified that the observed fluorescence changes were neither due to changes in SWCNT concentration nor chirality distribution (Fig. S1 and S2, ESI $\dagger$ ). The changes in fluorescence intensity were, thereby, assumed to be directly related to variations in the fluorescence QY of the complexes.

The QY of ssDNA-SWCNTs is believed to depend on a variety of factors. One such factor is DNA coverage on the SWCNT surface. The DNA wrapping reduces the amount of exposed SWCNT surface, shielding it from oxygen. ${ }^{26,27}$ Since oxygen induces fluorescence quenching, denser coverage can, therefore, yield increased fluorescence emission. ${ }^{26-28}$ The dependence of fluorescence intensity on SWCNT surface coverage also partially explains the chiralityand sequence-dependence of the fluorescence changes observed in this work. In agreement with these observations, previous studies have shown that the binding energy and wrapping angle of the DNA on the surface of the SWCNT can depend on the DNA sequence and the SWCNT chirality. ${ }^{29}$

The starting (GT) ${ }_{15}$-SWCNT complex is responsive towards certain neurotransmitters, including dopamine, which on addition results in a fluorescence increase. ${ }^{16}$ Dopamine detection assays were performed on the $(\mathrm{GT})_{15}$-SWCNTs and the mutant complexes in order to test whether the sensing capability was compromised post-mutation. A summary of these sensing responses is provided in Table S2 (ESI $\dagger$ ). Dopamine was found to increase fluorescence in a chirality-specific manner (Fig. 3a), with more pronounced responses for the $(8,6)$ chirality observed for both the $(\mathrm{GT})_{15^{-}}$and M13-SWCNTs ( $p$-value $\left.<0.05\right)$. No significant differences in dopamine response were observed between the $(\mathrm{GT})_{15}$ and mutant complexes for neither the $(9,4)$ chirality nor the integrated intensity. However, for the $(10,2)$ chirality, the M13-, M13-1-, and M13-6-SWCNTs showed overall stronger responses towards dopamine compared to the starting (GT) ${ }_{15}$-SWCNT complex ( $p$-value $<0.05)$. No response was observed for the $(\mathrm{GT})_{15^{-}}$or mutant complexes towards other neurotransmitters, including glutamic acid, $\gamma$-aminobutyric acid (GABA), acetylcholine, and glycine (Fig. 3a and Fig. S4, ESI $\dagger$ ), demonstrating that selectivity towards dopamine is retained post-mutation. A comparison of the calibration curves for the (GT) ${ }_{15^{-}}$and M13-SWCNT complexes (Fig. S5, ESI $\dagger$ ) confirmed that the mutant and original sensors are similarly able to detect $100 \mathrm{nM}$ of dopamine. a)

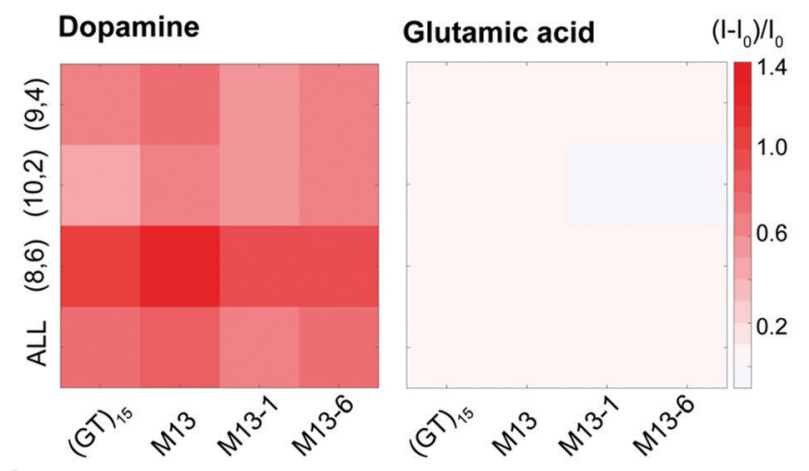

b)

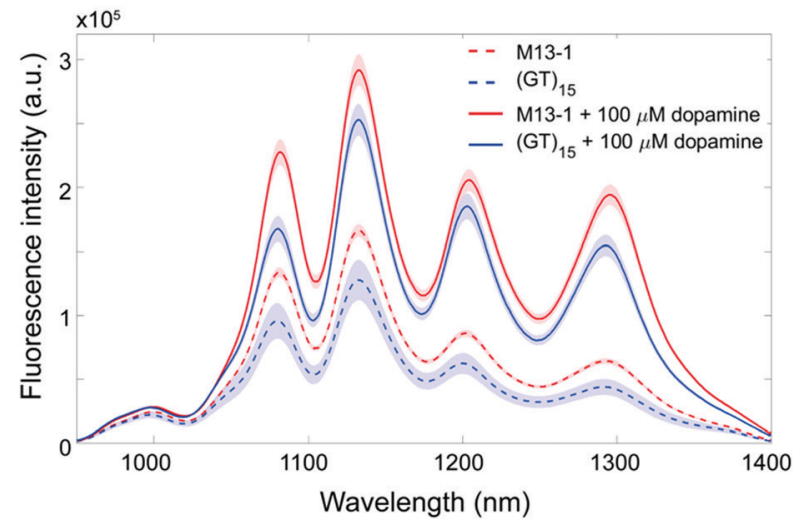

Fig. 3 Response of the sSDNA-SWCNT complexes to dopamine and glutamic acid (final analyte concentrations of $100 \mu \mathrm{M}$ ). (a) Response of the sSDNA-SWCNT sensors towards dopamine and glutamic acid as a function of the intensity of the $(9,4),(10,2)$, and $(8,6)$ chiralities as well as the integrated intensity under $745 \mathrm{~nm}$ excitation (labelled "ALL"). The response $I$ is normalised to the intensity before dopamine addition $I_{0}$. (b) Fluorescence spectra of the (GT) 15 -SWCNT (blue curves) and M13-1SWCNT (red curves) complexes before (dotted lines) and after (solid lines) dopamine addition. The shaded areas represent 95\% confidence intervals.

These results show that the mutant complexes demonstrate increased fluorescence intensities while simultaneously retaining the dopamine responsivity of the original sensor. In addition, the fluorescence enhancement of the mutant complexes relative to the $(\mathrm{GT})_{15}$-SWCNT complex is sustained even following the addition of dopamine (Fig. $3 \mathrm{~b}$ and Fig. S3, ESI $\dagger$ ). This improvement in the fluorescence intensity of these sensors, both before and after dopamine addition, can enable in vivo imaging at 
penetration depths up to $193 \mu \mathrm{m} \operatorname{deeper}^{30}$ (ESI $\dagger$ ), without compromising dopamine detection.

In this article, we evolved brighter ssDNA-SWCNT sensors through directed evolution of the DNA wrapping. While the reasons for the fluorescence intensity enhancement remain unclear, we demonstrate a guided approach that can be used to tune ssDNASWCNT properties in the absence of a defined structure-function relationship. Whereas previous work has focused on monitoring the response of a relatively small collection of random DNA sequences against a variety of analytes, we have instead focused on screening the performances of a larger library of mutants for a single characteristic, in this case fluorescence intensity. Although the observed SWCNT fluorescence increase is lower than other reported methods, ${ }^{26,31-35}$ the enhancement described herein was achieved without the addition of any exogenous compounds. This achievement is of particular importance for in vivo sensing applications since additives, such as reducing agents or nanoparticles, can lead to increased cytotoxicity and diminished sensitivity and/or selectivity of the sensor. ${ }^{26,31,32,36}$

While further enhancement may be achieved by refinements, such as screening even larger DNA libraries over additional evolution cycles, the results of this study aim to demonstrate the applicability of a new technique in navigating the fluorescence intensity landscape of ssDNA-SWCNTs. More generally, this work establishes a new, guided technique that can be applied not only to improve properties of existing optical SWCNT sensors, such as sensitivity and selectivity, but also to engineer new sensors with capabilities that are yet to be discovered.

We are thankful for financial support from the Swiss National Science Foundation Assistant Professor (AP) Energy Grant.

\section{Conflicts of interest}

There are no conflicts to declare.

\section{Notes and references}

1 Y. Wang, S. De and N. Yan, Chem. Commun., 2016, 52, 6210-6224.

2 A. Albanese, P. S. Tang and W. C. W. Chan, Annu. Rev. Biomed. Eng., 2012, 14, 1-16.

3 C. Kinnear, T. L. Moore, L. Rodriguez-Lorenzo, B. Rothen-Rutishauser and A. Petri-Fink, Chem. Rev., 2017, 117, 11476-11521.

4 S. Baskoutas and A. F. Terzis, J. Appl. Phys., 2006, 99, 013708.

5 J. C. Moore and F. H. Arnold, Nat. Biotechnol., 1996, 14, 303-308.

6 F. H. Arnold, Acc. Chem. Res., 1998, 31, 125-131.

7 M. S. Dresselhaus, G. Dresselhaus and P. C. Eklund, Science of Fullerenes and Carbon Nanotubes, 1996.

8 A. A. Boghossian, J. Zhang, P. W. Barone, N. F. Reuel, J. H. Kim, D. A. Heller, J. H. Ahn, A. J. Hilmer, A. Rwei, J. R. Arkalgud, C. T. Zhang and M. S. Strano, ChemSusChem, 2011, 4, 848-863.
9 S. Diao, J. L. Blackburn, G. Hong, A. L. Antaris, J. Chang, J. Z. Wu, B. Zhang, K. Cheng, C. J. Kuo and H. Dai, Angew. Chem., Int. Ed., 2015, 54, 14758-14762.

10 P. W. Barone and M. S. Strano, J. Diabetes Sci. Technol., 2009, 3, 242-252.

11 M. Zheng, A. Jagota, E. D. Semke, B. A. Diner, R. S. McLean, S. R. Lustig, R. E. Richardson and N. G. Tassi, Nat. Mater., 2003, 2, 338-342.

12 K. Umemura, Nanomaterials, 2015, 5, 321-350.

13 J. Zhang, A. A. Boghossian, P. W. Barone, A. Rwei, J. H. Kim, D. Lin, D. A. Heller, A. J. Hilmer, N. Nair, N. F. Reuel and M. S. Strano, J. Am. Chem. Soc., 2011, 133, 567-581.

14 A. Shankar, J. Mittal and A. Jagota, Langmuir, 2014, 30, 3176-3183.

15 G. Ao, J. K. Streit, J. A. Fagan and M. Zheng, J. Am. Chem. Soc., 2016, 138, 16677-16685.

16 S. Kruss, M. P. Landry, E. Vander Ende, B. M. A. Lima, N. F. Reuel, J. Zhang, J. Nelson, B. Mu, A. Hilmer and M. Strano, J. Am. Chem. Soc., 2014, 136, 713-724.

17 R. Haggenmueller, S. S. Rahatekar, J. A. Fagan, J. Chun, M. L. Becker, R. R. Naik, T. Krauss, L. Carlson, J. F. Kadla, P. C. Trulove, D. F. Fox, H. C. DeLong, Z. Fang, S. O. Kelley and J. W. Gilrnan, Langmuir, 2008, 24, 5070-5078.

18 P. V. Jena, M. M. Safaee, D. A. Heller and D. Roxbury, ACS Appl. Mater. Interfaces, 2017, 9, 21397-21405.

19 S. Kruss, D. P. Salem, L. Vukovic, B. Lima, E. Vander Ende, E. S. Boyden and M. S. Strano, PNAS, 2017, 114, 1789-1794.

20 J. P. Giraldo, M. P. Landry, S. Y. Kwak, R. M. Jain, M. H. Wong, N. M. Iverson, M. Ben-Naim and M. S. Strano, Small, 2015, 11, 3973-3984.

21 J. T. D. Bonis-O'Donnell, R. H. Page, A. G. Beyene, E. G. Tindall, I. R. McFarlane and M. P. Landry, Adv. Funct. Mater., 2017, 27, 1-10.

22 M. J. O'Connell, S. M. Bachilo, C. B. Huffman, V. C. Moore, M. S. Strano, E. H. Haroz, K. L. Rialon, P. J. Boul, W. H. Noon, C. Kittrell, J. Ma, R. H. Hauge, R. B. Weisman and R. E. Smalley, Science, 2002, 297, 593-596.

23 S. Lebedkin, K. Arnold, F. Hennrich, R. Krupke, B. Renker and M. M. Kappes, New J. Phys., 2003, 5, 140.

24 M. Jones, C. Engtrakul, W. K. Metzger, R. J. Ellingson, A. J. Nozik, M. J. Heben and G. Rumbles, Phys. Rev. B: Condens. Matter Mater. Phys., 2005, 71, 1-9.

25 J. Crochet, M. Clemens and T. Hertel, J. Am. Chem. Soc., 2007, 129, $8058-8059$.

26 D. P. Salem, X. Gong, A. T. Liu, V. B. Koman, J. Dong and M. S. Strano, J. Am. Chem. Soc., 2017, 139, 16791-16802.

27 A. J. Gillen, J. Kupis-Rozmysłowicz, C. Gigli, N. Schuergers and A. A. Boghossian, J. Phys. Chem. Lett., 2018, 9, 4336-4343.

28 Y. Zheng, S. M. Bachilo and R. B. Weisman, J. Phys. Chem. Lett., 2017, 8, 1952-1955.

29 M. L. Mayo, Z. Q. Chen and S. V. Kilina, J. Phys. Chem. Lett., 2012, 3, $2790-2797$.

30 M. A. Lee, F. T. Nguyen, K. Scott, N. Y. L. Chan, N. A. Bakh, K. K. Jones, C. Pham, P. Garcia-Salinas, D. Garcia-Parraga, A. Fahlman, V. Marco, V. B. Koman, R. J. Oliver, L. W. Hopkins, C. Rubio, R. P. Wilson, M. G. Meekan, C. M. Duarte and M. S. Strano, ACS Sens., 2019, 4, 32-43.

31 F. Sen, A. A. Boghossian, S. Sen, Z. W. Ulissi, J. Zhang and M. S. Strano, ACS Nano, 2012, 6, 10632-10645.

32 A. J. Lee, X. Wang, L. J. Carlson, J. a. Smyder, B. Loesch, X. Tu, M. Zheng and T. D. Krauss, Nano Lett., 2011, 11, 1636-1640.

33 E. Polo and S. Kruss, J. Phys. Chem. C, 2016, 120, 3061-3070.

34 J. Yang, Q. Zhao, M. Lyu, Z. Zhang, X. Wang, M. Wang, Z. Gao and Y. Li, Small, 2016, 12, 3164-3171.

35 T. Shiraki, T. Shiraishi, G. Juhász and N. Nakashima, Sci. Rep., 2016, 6, 1-9.

36 K. D. Held and D. C. Melder, Radiat. Res., 1987, 112, 544-554. 